\title{
Severe oxidative stress in sickle cell disease patients with uncomplicated Plasmodium falciparum malaria in Kampala, Uganda
}

\author{
Saad Mahjub Atiku, Nabukeera Louise and Dennis M. Kasozi (D)
}

\begin{abstract}
Background: Oxidative stress plays a vital role in the pathogenesis of both Sickle Cell Disease (SCD) and Plasmodium falciparum malaria. However, there are limited studies on the effect of $P$. falciparum malaria infection on oxidative stress in SCD patients.

Methods: A cross-sectional study was undertaken to compare levels of biomarkers of oxidative stress in isolates from SCD patients with uncomplicated P.falciparum malaria. The biomarkers namely: malondialdehyde (MDA), reduced glutathione (GSH), catalase (CAT) and glutathione peroxidase (GPX) were determined in plasma samples from SCD malaria positive, malaria positive, SCD malaria negative and healthy control participants. The genetic diversity of P.falciparum was determined by nested polymerase chain reaction of merozoite surface protein-2 (MSP2) gene.
\end{abstract}

Results: Out of 207 participants, 54 (26\%) were SCD malaria positive, 51 (24\%) malaria positive, 51 (24\%) SCD controls and 51 (24\%) healthy control individuals. The mean concentration of MDA was significantly higher in SCD malaria positive than SCD controls $(P<0.0001)$. In contrast, the mean concentration of GSH $(P<0.0001)$ and GPx $(P<0.0001)$ were significantly lower in SCD malaria than SCD controls. Although not significantly different, the mean concentration of MDA was higher $(P=0.0478)$, but the geometric mean parasite density $(P=0.2430)$ and multiplicity of infection $(P=0.3478$ ) were lower in SCD malaria samples than in malaria samples. The most prevalent MSP2 allelic family was IC3D7 in SCD malaria (72\%) and Malaria (76\%) samples. The biomarkers of oxidative stress were not significantly different between IC3D7 and FC27 allelic families of MSP2.

Conclusion: We identified severe oxidative stress in Sickle cell disease patients with uncomplicated P.falciparum malaria.

Keywords: Sickle cell disease, Malaria, Biomarkers of oxidative stress, Malondialdehyde, Catalase, Uganda

\section{Background}

Sickle cell disease (SCD) and Malaria are co-endemic in Africa. SCD is a hemoglobinopathy caused by a single mutation in the $\beta$-globin chain resulting in the substitution of valine for glutamic acid at the sixth amino acid position [1]. Worldwide, an estimated 312,000 children are born with SCD annually $75 \%$ of these in Sub Saharan Africa [2]. In Uganda it is estimated that 15,000 babies are born with SCD and of these $50-80 \%$ die annually

\footnotetext{
* Correspondence: dkasozi@cns.mak.ac.ug; dmkkasozi@gmail.com
Department of Biochemistry and Sports Science, School of Biosciences,
College of Natural Sciences, Makerere University Kampala, P.O. BOX 706

* Correspondence: dkasozi@cns.mak.ac.ug; dmkkasozi@gmail.com
Department of Biochemistry and Sports Science, School of Biosciences,
College of Natural Sciences, Makerere University Kampala, P.O. BOX 7062

* Correspondence: dkasozi@cns.mak.ac.ug; dmkkasozi@gmail.com
Department of Biochemistry and Sports Science, School of Biosciences,
College of Natural Sciences, Makerere University Kampala, P.O. BOX 7062 Kampala, Uganda
}

(c) The Author(s). 2019 Open Access This article is distributed under the terms of the Creative Commons Attribution 4.0 International License (http//creativecommons.org/licenses/by/40/) which permits unrestricted use, distribution, and reproduction in any medium, provided you give appropriate credit to the original author(s) and the source, provide a link to the Creative Commons license, and indicate if changes were made. The Creative Commons Public Domain Dedication waiver (http://creativecommons.org/publicdomain/zero/1.0/) applies to the data made available in this article, unless otherwise stated.

before 5 years [3]. With Uganda having the third highest number of Plasmodium falciparum infections in subSaharan Africa [4], malaria is considered a significant cause of this SCD mortality [5].

Oxidative stress is plays a vital role in the pathophysiology of both SCD [6] and malaria [7]. In SCD, oxidative stress results from increased intravascular hemolysis, ischemia-reperfusion injury, and chronic inflammation [6]. In response to P.falciparum infection, phagocytes generate large amounts of reactive oxygen species (ROS) and reactive nitrogen species (RNS) causing an imbalance between oxidizing species and antioxidants [7]. This imbalance triggers oxidative stress, which is an 
important mechanism of human hosts in response to infection and can lead to the death of the parasites [8] although it may also lead to cell and tissue damage [9]. The host enzymatic and nonenzymatic antioxidant cellular defense system plays a key role in protecting biological systems from ROS by regulating the production of free radicals and their metabolites [10]. The primary antioxidant enzymes against superoxide radicals include superoxide dismutase (SOD), catalase (CAT), and glutathione peroxidase (GPx) [10]. The major non-enzymatic antioxidant cellular defense systems comprise glutathione, vitamin C and Vitamin E [10]. Limited studies have been undertaken on oxidative stress in SCD patients in Sub-Saharan Africa. Malondialdehyde (MDA) which is a product of lipid peroxidation is an important marker in the evaluation of oxidative stress $[9,10]$. Recently, a study in Cameroon showed increased MDA in SCD patients compared to healthy individuals [11]. However, no study has evaluated oxidative stress in SCD patients with P.falciparum malaria in Uganda.

In addition, to date there is very limited information on the impact of oxidative stress on the genetic diversity of P.falciparum. Strains of P.falciparum are distinguished by highly polymorphic markers, in particular the merozoite surface protein 2 (MSP2) [12]. Recently, significant disparity in allele frequencies of K1, MAD20, RO33 of MSP 1 were found in SCD patients with malaria in Nigeria [13]. Yet, by far MSP2 is more polymorphic and has the higher heterozygosity values than MSP1 [12]. However, no studies have been undertaken on the distribution of IC3D7 and FC27 the allelic families of MSP2 in SCD patients in Sub Sahara Africa.

It is important to evaluate the oxidative status of SCD patients with P.falciparum malaria. This evidence would be relevant in the improved management of malaria in SCD. The aim of this study was to characterize oxidative stress in SCD patients with P.falciparum malaria.

\section{Methods}

\section{Study design}

This was a cross-sectional study done between November 2015 and April 2016. Ethical approval was obtained from Mulago National Referral Hospital Ethics Committee. The inclusion criteria were: blood samples of SCD patients confirmed positive for P. falciparum, SCD patients who were malaria negative, malaria positive and healthy controls. SCD was confirmed by $\mathrm{Hb}$ electrophoresis on cellulose acetate membrane. The exclusion criteria were: Samples from patients with chronic and complicated conditions including severe or complicated malaria, severe anaemia $(\mathrm{Hb}<7 \mathrm{~g} / \mathrm{dl})$ and other infections like HIV and on any treatment were excluded. Samples were obtained from participants after informed consent from their parents or guardians. All patients were treated as per the national antimalarial guidelines. To diagnose P.falciparum by microscopy, thick blood films of every participant were made and stained using $10 \%$ Giesma stain. The parasite density was expressed as number of parasites per microlitre $(\mu \mathrm{L})$ of blood, assuming a WBC count of 8000 leucocytes per $\mu \mathrm{L}$ of blood.

\section{Blood sample collection}

Venous blood $(3 \mathrm{~mL})$ was collected from each participant into a purple top tube containing Ethylene Diamine Tetra Acetate (EDTA). All the blood samples were centrifuged at X3000rpm for $10 \mathrm{~min}$. The plasma if not analyzed immediately was stored for not longer than $48 \mathrm{~h}$ in a freezer $\left(-20^{\circ} \mathrm{C}\right)$ for the MDA and GSH assays. A total of 207 samples were collected from Mulago Hospital, Kampala Uganda. Out of these 105 samples were from Sickle Cell Clinic of Mulago hospital, 54 were P.falciparum malaria positive and 51 negative (sickle cell control). Fifty one P.falciparum malaria positive were obtained from Malaria Lab at Assessment Centre of Mulago Hospital. Fifty one samples for Healthy control group were obtained from the Nakasero Blood Bank and tested within $48 \mathrm{~h}$ after collection. In addition, RBCs were lysed with ice cold water and centrifuged at 5000 $\mathrm{rpm}$ for $10 \mathrm{~min}$ at $4{ }^{\circ} \mathrm{C}$ to obtain the clear lysate that was used for the CAT and GPx assays. The analysis for the biomarkers of oxidative stress was done at the Biochemistry Department, Makerere University.

\section{Preparation of dry blood spots (DBS)}

P. falciparum positive Blood samples $(50 \mu \mathrm{L})$ were placed on 903 filter papers (Fitzco, Minneapolis, MN) labeled, air dried and then placed in plastic bags. The DBS were stored at ambient temperature for MSP 2 genotyping.

\section{Malondialdehyde (MDA)}

MDA was determined using the Thiobarbituric acid reactive substances (TBARS) assay which is based on the reaction between MDA and 2-thiobarbituric acid, a chromogenic reagent to form a chromophore with absorbance maximum at $532 \mathrm{~nm}$ [14]. In the assay, $300 \mu \mathrm{l}$ of the sample plasma was mixed with $200 \mu \mathrm{L}$ of Trichloro acetic acid (TCA)/ Thiobarbituric acid (TBA) solution containing $15 \mathrm{~g}$ of TCA and $0.67 \mathrm{~g}$ TBA in $50 \mathrm{ml}$ of $0.25 \mathrm{M} \mathrm{HCl}$. Next $300 \mu \mathrm{l}$ of $1 \mathrm{M} \mathrm{HCl}$ was added to this mixture and then heated at $100^{\circ} \mathrm{C}$ for $60 \mathrm{~min}$. The mixture was cooled on ice for $20 \mathrm{~min}$ and centrifuged at $3000 \mathrm{rpm}$ for $2 \mathrm{~min}$. To $350 \mu \mathrm{l}$ of the supernatant was then added $300 \mu \mathrm{l}$ of $3 \mathrm{M} \mathrm{NaOH}$ and vortexed. The mixture was spinned again at $1300 \mathrm{rpm}$ for $1 \mathrm{~min}$ before reading the absorbance at $540 \mathrm{~nm}$ within $10 \mathrm{~min}$. 


\section{Level of the reduced glutathione (GSH)}

The determination of Glutathione levels in the plasma was performed as described [15]. The assay is based on the oxidation of GSH by 2-nitrobenzoic acid (DTNB) to form the yellow chromophore 5'-thio-2-nitrobenzoic acid (TNB), with absorbance at $412 \mathrm{~nm}$. Briefly, $200 \mu \mathrm{L}$ of $10 \%$ solution of TCA was added into $1.5 \mathrm{ml}$ Eppendorf tube followed by $100 \mu \mathrm{L}$ of freshly thawed plasma. The mixture was then vortexed, centrifuged at $4000 \mathrm{x} \mathrm{g}$ for $10 \mathrm{~min}$ and the supernatant separated. To $200 \mu \mathrm{L}$ of the supernatant $700 \mu \mathrm{L}$ of $400 \mathrm{mM}$ Tris- $\mathrm{HCl}$ buffer, $\mathrm{pH}$ 8.9 was added followed by $100 \mu \mathrm{L}$ of $2.5 \mathrm{mM}$ DTNB dissolved in $40 \mathrm{mM}$ Tris- $\mathrm{HCl}$ buffer, $\mathrm{pH} 8.9$ and the mixture was then incubated at room temperature for 10 min. The optical densities (O.Ds) of the samples/standards and the blank (made of DTNB instead of sample/ standard) at $412 \mathrm{~nm}$ were measured. The O.Ds were then used to determine the concentration of GSH using the calibration graph of the standards.

\section{Activity of glutathione peroxidase (GPx)}

This was measured indirectly using a modification of Ursini et al., [16] where $100 \mu \mathrm{l}$ of $2 \mathrm{mM} \mathrm{GSH}$ solution was added to $150 \mu \mathrm{l}$ of $0.1 \mathrm{M}$ phosphate buffer $\mathrm{pH}$ 7.4. Then $50 \mu \mathrm{l}$ of $10 \mathrm{mM}$ sodium azide and $1 \mathrm{mM} \mathrm{H}_{2} \mathrm{O}_{2}$ was added to this mixture followed by $150 \mu \mathrm{l}$ of the sample lysate and the mixture was then vortexed. A blank made of $400 \mu \mathrm{l}, 50 \mu \mathrm{l}$ and $50 \mu \mathrm{l}$ of $0.1 \mathrm{M}$ phosphate buffer $\mathrm{pH}$ 7.4, $10 \mathrm{mM}$ sodium azide and $1 \mathrm{mM} \mathrm{H}_{2} \mathrm{O}_{2}$ respectively while the reference standard consist of $300 \mu \mathrm{l}, 100 \mu \mathrm{l}$, $50 \mu \mathrm{l}$ and $50 \mu \mathrm{l}$ of $0.1 \mathrm{M}$ phosphate buffer $\mathrm{pH} 7.4,2 \mathrm{mM}$ GSH solution, $10 \mathrm{mM}$ sodium azide and $1 \mathrm{mM} \mathrm{H}_{2} \mathrm{O}_{2}$ respectively. The mixtures were then incubated at $37^{\circ} \mathrm{C}$ for $15 \mathrm{~min}$ in a water bath, $250 \mu \mathrm{l}$ of $5 \% \mathrm{TCA}$ was added and the mixture spinned at X2000rpm for $5 \mathrm{~min}$. Next $25 \mu \mathrm{l}$ of the supernatant was then transferred into a 96 well plate containing $50 \mu \mathrm{l}$ of the buffer and $175 \mu \mathrm{l}$ of DTNB. The plate was shaken for $1 \mathrm{~min}$ and absorbance read at $405 \mathrm{~nm}$ using an ELISA plate reader.

\section{Activity of catalase}

The activity of catalase was determined using a modification of a colorimetric assay as described [17] which determines the $\mathrm{H}_{2} \mathrm{O}_{2}$ content of erythrocyte lysates. Briefly, $200 \mu \mathrm{l}$ of $0.01 \mathrm{M}$ phosphate buffer was added to $300 \mu \mathrm{l}$ of $0.2 \mathrm{M}$ hydrogen peroxide and to this mixture $20 \mu \mathrm{l}$ of diluted sample lysate (1:10 with $0.01 \mathrm{M}$ phosphate buffer $\mathrm{pH}$ 7.0) was added. This mixture was then swirled, incubated at room temperature for $1 \mathrm{~min}$ and $500 \mu \mathrm{l}$ of $5 \%$ solution of $\mathrm{K}_{2} \mathrm{Cr}_{2} \mathrm{O}_{7}$ with glacial acetic acid (1,3 by volume) added. The mixture was vortexed and incubated at $100^{\circ} \mathrm{C}$ for $10 \mathrm{~min}$ before reading absorbance at $580 \mathrm{~nm}$.

\section{Extraction of DNA}

Total genomic DNA was extracted from dried blood spots (DBS) on paper cards (Fitzco, Minneapolis, MN) by punching $3 \mathrm{~dB}$ into a $1.5 \mathrm{ml}$ tube. To this $300 \mu \mathrm{L}$ of methanol was added to completely immerse the DBS and the content to be incubated at room temperature for $15 \mathrm{~min}$. The methanol was then discarded carefully and the retained DBS dried in the tubes for 15 min. 100 $\mu \mathrm{L}$ of distilled water was added to the DBS and the content heated at temperatures of between 95 and $100^{\circ} \mathrm{C}$ for $15 \mathrm{~min}$ while occasionally vortexing to increase yield. The extracted DNA was then transferred into a $25 \mu \mathrm{L}$ PCR reaction tube or frozen for later use in analysis of the MSP2 gene.

\section{Genotyping MSP2 of P.falciparum isolates}

PCR was a two-step reaction involving a primary and nested PCR reaction in which amplification of the entire $m s p 2$ gene was followed by targeting the allelic families of IC/3D7 and FC27 [12]. The sequence of the oligonucleotide primers and genomic DNA from cloned laboratory strains which were used as positive controls are shown in Table 1 . Both primary and nested PCR was used to amplify the polymorphic repetitive region (block 3) of $m s p 2$ where in the primary reaction, primer pairs corresponding to conserved sequences spanning the polymorphic region of the $m s p 2$ genes was used and in the second nested reactions, specific primer pair was then used to determine the presence of allelic variants from the FC27 and the IC/3D7 families of the msp2 repeats.

Amplifications was performed in Thermocycler (Biometra, T3000) with a total volume of the reaction to be $25 \mu \mathrm{L}$ which was composed of $23 \mu \mathrm{L}$ of a master mix ( $160.5 \mu \mathrm{L}$ of distilled $\mathrm{H}_{2} \mathrm{O}, 5 \mu \mathrm{L}$ each of primer 1 and primer $2,25 \mu \mathrm{L}$ of buffer, $25 \mu \mathrm{L}$ of dNTPs, $7.5 \mu \mathrm{L}$ of $\mathrm{MgCl}_{2}$ and $2 \mu \mathrm{L}$ of Taq Polymerase) and $2 \mu \mathrm{L}$ of the samples and the controls. Thus $23 \mu \mathrm{L}$ of the master mix was added to each well of a standard PCR tube corresponding to a sample or a control followed by $2 \mu \mathrm{L}$ of the sample or control and pressure applied for tight sealing. The thermocycler which was programmed to perform the primary PCR amplification of $m s p 2$ genes as follows; an initial step of $95^{\circ} \mathrm{C}$ for $5 \mathrm{~min}$ followed by 30 cycles of $95^{\circ} \mathrm{C}$ for $1 \mathrm{~min}, 55^{\circ} \mathrm{C}$ for $2 \mathrm{~min}, 72^{\circ} \mathrm{C}$ for 2 min, and a final extension of $72^{\circ} \mathrm{C}$ for $5 \mathrm{~min}$..

To identify the positive samples, the nested msp 2 amplification product was separated in a $2 \%$ ethidium bromide-stained agarose gel. Briefly $3 \mu \mathrm{L}$ of loading dye was mixed with $15 \mu \mathrm{L}$ of the PCR product and carefully loaded into an appropriate well on a gel. The first and last wells were used for the DNA size standards (50 bp). PCR products were loaded between the first and last wells. Agarose gel electrophoresis was carried out for 30 
Table 1 Primers sequences and annealing temperature for nested PCR of MSP2

\begin{tabular}{|c|c|c|c|c|c|}
\hline PCR round & Primer name. & Primer Sequence. $\left(5^{\prime} \rightarrow 3^{\prime}\right)$ & Annealing temp $\left({ }^{\circ} \mathrm{C}\right)$ & Positive Control & Allelic Family \\
\hline \multirow[t]{2}{*}{ Primary } & 1 & ATGAAGGTAATTAAAACATTGTCTATTATA & 55 & & \\
\hline & 4 & ATATGGCAAAAGATAAAACAAGTGTTGCTG & 55 & & \\
\hline \multirow[t]{4}{*}{ Secondary } & A1 & GCAGAAAGTAAGCCTTCTACTGGTGCT & 55 & 3D7 & IC3D7 \\
\hline & A2 & GATTTGTTCGGCATTATTATGA & 55 & & \\
\hline & B1 & GCAAATGAAGGTTCTAATACTAATAG & 55 & HB3 & $\mathrm{FC} 27$ \\
\hline & B1 & GCTTTGGGTCCTTCTTCAGTTGATTC & 55 & & \\
\hline
\end{tabular}

$P C R$ Polymerase chain reaction, MSP2 Merozoite surface protein 2

min at $85-90 \mathrm{~V}$. Once the gel had run sufficiently, it was photographed digitally using UV light and saved in a format compatible with GelCompar II, a gel processing software for band sizing and molecular weight calculations. The ratio of the number of total fragments to the number of positive samples was taken as the MOI $[18,19]$.

\section{Data analysis}

The results were analyzed using GraphPad Prism 5.03 software. One way analysis of variance (ANOVA) was used to assess significant differences between the groups. The linear association between parasite density and biomarkers of oxidative stress were analyzed by Pearson correlation coefficient. Statistical significance was defined as $P<0.05$.

\section{Results}

\section{Baseline characteristics}

Out of 207 participants, 54 (26\%) were SCD malaria positive, 51 (24\%) malaria positive, 51 (24\%) SCD malaria negative and 51 (24\%) healthy control individuals. The mean age (years) of SCD malaria positive, malaria positive, SCD malaria negative and healthy control individuals were 14.1 (SD 10.2), 12.6 (SD 12.2), 14.8 (SD13.6) and 22 (SD 7.2) respectively. The percentage of females in SCD malaria positive was $44.4 \%$ (24/54), malaria positive $47.1 \%(24 / 51)$, SCD malaria negative $45.1 \%$ (23/51) and healthy control individuals 54.9\% (28/51). The mean hemoglobin concentration $(\mathrm{g} / \mathrm{dL})$ of SCD malaria positive, malaria positive, SCD controls and healthy control individuals were 8.4 (SD 1.9), 12.9 (SD 1.6), 9.2 (SD 1.5) and 14.2 (SD 1.4) respectively. The geometric mean parasite density (GMPD) was lower in SCD malaria (16,800, 95\%CI 12,131-23,266 parasites/ $\mu \mathrm{L})$ than in the malaria samples $(19,306,95 \% \mathrm{CI} 16,706-$ 22,310 parasites $/ \mu \mathrm{L})$ but the difference was not significant (Unpaired t test, $P=0.2430$ ).

\section{Markers of oxidative stress in sickle cell malaria patients and control groups}

The mean levels of markers of oxidative stress namely MDA, GSH, GPx and CAT are shown in Table 2. The variations in the levels of markers of oxidative stress are shown in Fig. 1. The mean concentration of MDA was significantly higher in isolates from SCD malaria positive compared to SCD control group $(P<0.0001)$. By contrast, mean levels of GPx $(P<0.0001)$ and CAT $(P=$ $0.0022)$ were significantly higher in SCD control group than SCD malaria.

Although the mean level of GSH was also higher in SCD control group than SCD malaria, the difference was not statistically significant $(P=0.0933)$. The mean concentration of MDA was significantly higher in isolates from malaria positive compared to healthy control group (Table $1, P<0.0001$ ). Conversely, mean levels of GSH $(P<0.0001)$, GPx $(P<0.0001)$ and CAT $(\mathrm{P}<0.0001)$ were significantly higher in the healthy control group than in the malaria group.

There was no significant difference $(P=0.0905)$ in mean level of MDA between SCD control group and the healthy control group. On the contrary, mean levels of GSH $(P<0.0001)$, GPx $(P<0.0001)$ and CAT $(P<$ 0.0001 ) were significantly higher in healthy control group than the SCD control group. Although not

Table 2 Markers of oxidative stress

\begin{tabular}{lllll}
\hline Group & MDA $\pm S D(\mu M)$ & GSH \pm SD $(\mathrm{mM})$ & GPx \pm SD $($ Units/ml $)$ & CAT \pm SD (Units) \\
\hline SCD malaria & $1.58 \pm 0.456$ & $0.726 \pm 0.028$ & $0.344 \pm 0.0802$ & $0.355 \pm 0.156$ \\
Malaria & $1.39 \pm 0.325$ & $0.767 \pm 0.031$ & $0.412 \pm 0.0962$ & $0.383 \pm 0.164$ \\
SCD control & $0.81 \pm 0.300$ & $0.766 \pm 0.063$ & $0.530 \pm 0.0115$ & $0.436 \pm 0.129$ \\
Healthy & $0.88 \pm 0.277$ & $0.929 \pm 0.083$ & $0.615 \pm 0.0824$ & $0.562 \pm 0.108$ \\
$P$-value & $0.0001^{* *}$ & $0.0001^{* *}$ & $0.0001^{* *}$ & $0.0001^{* *}$ \\
\hline
\end{tabular}

MDA Malondialdehyde, GS Reduced Glutathione, CAT Catalase, GPX Glutathione peroxidase **Statistically significant, ${ }^{\text {a ANOVA }}$ 

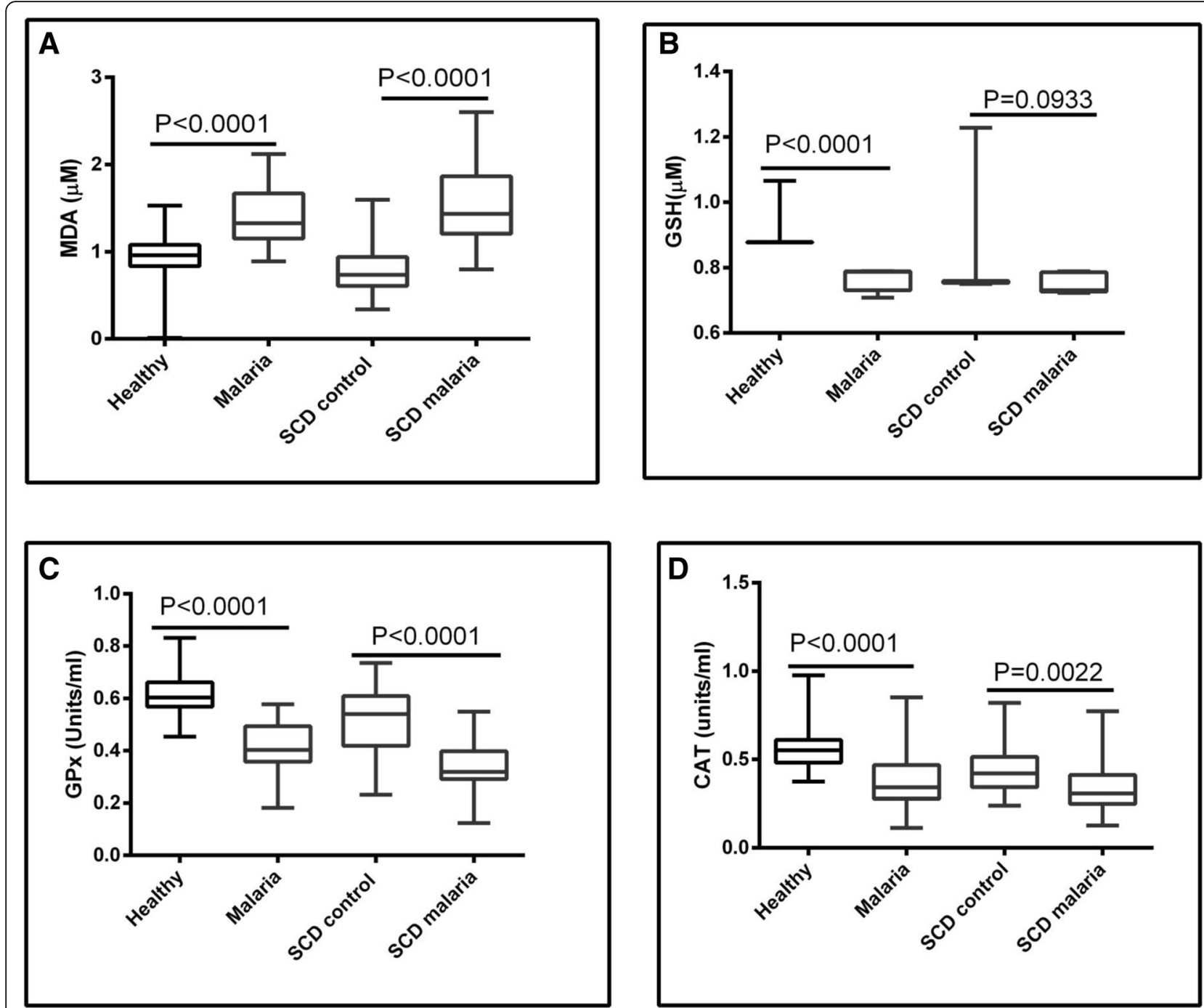

Fig. 1 Variation in Biomarkers of Oxidative stress. The concentration of MDA (a) increased but the concentration of GSH (b) and activity GPx (c) as well as that of CAT (d) decreased between healthy control and malaria as well as between the SCD control and SCD malaria group. The mean MDA, GSH, GPx and CAT were significantly different (ANOVA, $P$ < 0.001). MDA: Malondialdehyde; GSH: reduced glutathione; GPx: glutathione peroxidase; CAT: Catalase

significantly different, the mean level of MDA was also higher in SCD malaria group than malaria group $(P=$ 0.0478). In contrast, mean levels of GSH $(P=0.0052)$, and $\operatorname{GPx}(P=0.0002)$ were significantly higher in malaria group than the SCD malaria group. The mean level of CAT was also higher in malaria group than SCD malaria group, but the difference was not statistically significant $(P=0.3085)$.

\section{Genetic diversity, allelic frequency and multiplicity of infection}

Out of 50 isolates analysed, $25(50 \%)$ malaria positive and 25 (50\%) SCD malaria positive isolates were successfully genotyped for the detection of MSP2 alleles. The fragement sizes for IC3D7 and FC27 in SCD malaria and malaria samples are shown in Fig. 2. The most prevalent MSP2 allelic family was IC3D7 in SCD malaria (72\%) and Malaria (76\%) samples. For the SCD malaria positive isolates, $24 \%$ were positive for IC3D7 allelic family, 20\% for the FC27 allelic family, 48\% for both allelic families. In the P.falciparum malaria positive isolates, $36 \%$ were positive for the 3D7 allelic family, 20\% for the FC27 allelic family, $40 \%$ for both allelic families. In the SCD malaria positive isolates, 14 different alleles were identified with 7 alleles belonging to each of the allelic families of IC3D7 and FC27. In the malaria positive isolates, 18 alleles in total were identified with 10 for the 3D7/IC family (50-600 bp) and 8 for the FC27 family (150-550 bp) The Multiplicity of infection (MOI) was lower in the SCD malaria positive isolates (1.67) than in the malaria 


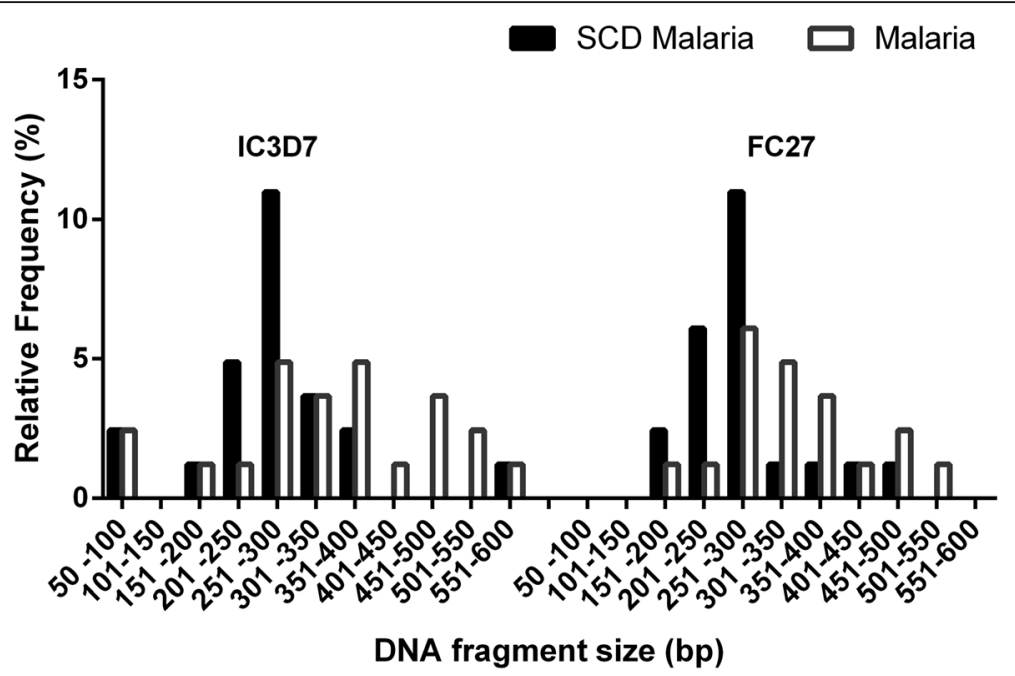

Fig. 2 Allelic families of MSP2. Comparison of the distribution of the IC/3D7 and FC27 allelic families between isolates from malaria and SCD malaria patients

positive (1.75) isolates but the difference was not statistically different $(P=0.3478)$.

Parasite density and biomakers of oxidative stress

Table 3 shows the Pearson correlation coefficents between parasite density and levels of biomarkers of oxidative stress. There were no significant correlations between parasite density and MDA, activity of GPx and CAT in SCD patients with malaria except for GSH with parasite density (Fig. 3). In the normal patients with P. falciparum malaria, parasite density with MDA or GPx had a positive correlation which was not statistically significant. However, GSH and CAT had negative correlation but was also not statistically significant.

Table 3 Correlation of Parasite Density with Biomarkers of oxidative stress

\begin{tabular}{|c|c|c|c|c|}
\hline & $\mathrm{MDA}(\mu \mathrm{M})$ & GSH $(\mu \mathrm{M})$ & GPx (Units/ml) & CAT (U/mg prot) \\
\hline \multicolumn{5}{|l|}{ SCD malaria } \\
\hline$n$ & 51 & 51 & 51 & 51 \\
\hline Pearson $r$ & 0.07110 & -0.5123 & -0.02336 & 0.1780 \\
\hline$P$ value & 0.6237 & 0.0001 & 0.8721 & 0.2162 \\
\hline$R^{2}$ & 0.005055 & 0.2625 & 0.0005455 & 0.03168 \\
\hline \multicolumn{5}{|l|}{ Malaria } \\
\hline$n$ & 50 & 50 & 50 & 50 \\
\hline Pearson $r$ & 0.1206 & -0.1142 & 0.1198 & -0.01226 \\
\hline$P$ value & 0.4040 & 0.4295 & 0.4074 & 0.9327 \\
\hline$R^{2}$ & 0.01455 & 0.01305 & 0.01435 & 0.0001503 \\
\hline
\end{tabular}

Pearson's $\mathbf{r}$ values from scatter plots. With $n=50$, MDA is malondialdehyde; $\mathrm{GSH}$ is reduced glutathione; GPx is Glutathione peroxidase; CAT is catalase
Malondialdehyde and other biomarkers of oxidative stress

Table 4 shows pearson correlation coefficents between MDA and other biomarkers of oxidative stress. In the SCD malaria group, malaria group and SCD control group, the correlations between MDA with GPx were positive but not statistically significant. In constrast, in the healthy control group there was no correlation between MDA with GPx. In all groups, there were no correlations between MDA with GSH and CAT.

Biomarkers of oxidative stress and MSP2 alleles

No significant differences were observed between MDA, GSH, GPx and CAT between IC3D7 and FC27

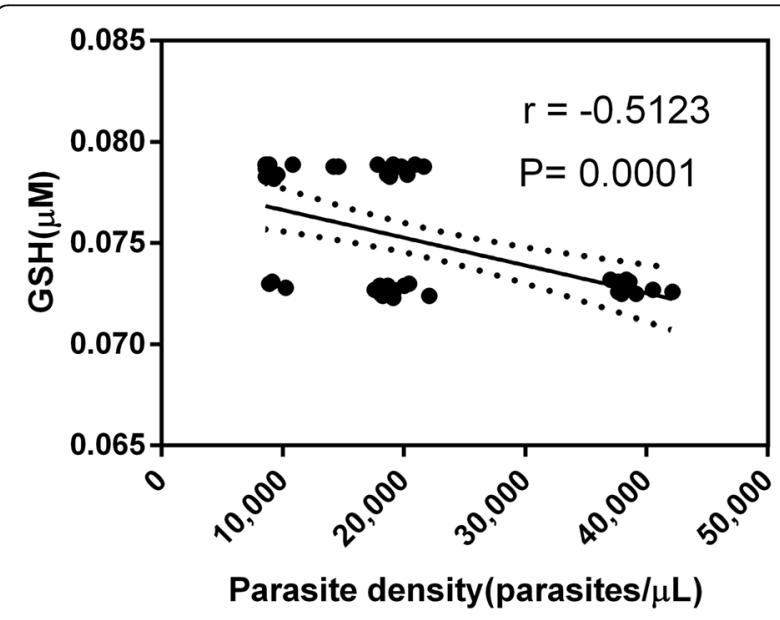

Fig. 3 Correlation between Parasite density and Glutathione. In the SCD patients with malaria, the correlation between parasite density (parasites/ $\mu \mathrm{L}$ ) and glutathione $(\mu \mathrm{M})$ was significant (Pearson's $r=-0.5123, P=0.0001)$ 
Table $4 \mathrm{MDA}$ and other Biomarkers of Oxidative stress

\begin{tabular}{llll}
\hline Biomarker & GSH & GPx & CAT \\
\hline SCD Malaria $n$ & 51 & 51 & 51 \\
Pearson $r$ & -0.2500 & 0.2893 & -0.1456 \\
$P$ value (two-tailed) & 0.0769 & 0.0395 & 0.3081 \\
$R^{2}$ & 0.06249 & 0.08369 & 0.02119 \\
Malaria & & & \\
Pearson r & 0.01729 & 0.07464 & 0.1865 \\
$P$ value (two-tailed) & 0.9013 & 0.5917 & 0.1769 \\
$R^{2}$ & 0.0002988 & 0.005571 & 0.03479 \\
SCD control & & & \\
Pearson r & -0.05931 & -0.3836 & 0.07885 \\
$P$ value (two-tailed) & 0.6793 & 0.0055 & 0.5823 \\
$R^{2}$ & 0.003518 & 0.1472 & 0.006217 \\
Healthy control & & & \\
Pearson r & 0.01596 & 0.0278 & -0.01898 \\
$P$ value (two-tailed) & 0.9115 & 0.8464 & 0.8948 \\
$R^{2}$ & 0.0002547 & 0.0007729 & 0.0003601 \\
\hline
\end{tabular}

MDA malondialdehyde, GSH reduced glutathione, GPX Glutathione peroxidase (a negative $r$ value that is significant), CAT catalase

in SCD malaria and malaria samples. Table 5 shows the relationship between the oxidative stress biomarkers and MSP2 allele families.

\section{Discussion}

Our study for the first time, characterized significantly elevated oxidative stress in patients having the SCD with uncomplicated P.falciparum malaria. This work assessed biomarkers of oxidative stress including malondialdehyde (MDA), reduced glutathione (GSH), glutathione peroxidase (GPx) and catalase (CAT) in isolates from SCD patients with P.falciparum malaria.
In SCD, the rate of polymerization of sickled hemoglobin $(\mathrm{HbS})$ increases with increase in its concentration. This increase in polymerization results into elevated hemolysis and hence the release of cell-free heme released after autoxidation [19]. During the reperfusion period, heme auto-oxidizes in the presence of oxygen into methemoglobin and superoxide. Whereas the normal hemoglobin (HbA) can convert them to harmless byproducts, the $\mathrm{HbS}$ produces $\mathrm{H}_{2} \mathrm{O}_{2}$ which when exposed to methemoglobin, decomposes hemoglobin to form ferrous $\left(\mathrm{Fe}^{2+}\right)$ [20]. Hydrogen peroxide then reacts with the ferrous $\left(\mathrm{Fe}^{2+}\right)$ formed to liberate the more reactive and harmful radical of hydroxyl $(\bullet \mathrm{OH})$ [20]. This implies that $\mathrm{HbS}$ liberates more concentrations of superoxide, $\mathrm{H}_{2} \mathrm{O}_{2}$, and $\bullet \mathrm{OH}$ than $\mathrm{HbA}$ and hence increased oxidative stress.

The current study showed higher concentration of MDA in the malaria positive samples than the negative samples indicating increased oxidative stress. These findings are in agreement with previous results $[21,22]$. These results may be explained by the fact that the malaria parasite is capable of generating reactive oxygen species within the erythrocytes $[6,7]$. Furthermore, increased oxidative stress may be explained as arising from the ROS produced from immune activation against the malarial parasite an antimicrobial action [23].

The difference in mean level of MDA between SCD control group and healthy control group, was not statistically significant $(P=0.0905)$. A previous study in Cameroon showed that MDA in SCD patients was significantly higher than in healthy individuals [11]. This discrepancy in results may be due hydroxyurea medication used by the sickle cell disease patients. Hydroxyurea has been shown have antioxidant property of decreasing the levels of lipid peroxidation [24].

GSH is a vital antioxidant in redox homeostasis. The increased oxidative stress in both the Sickle cell disease

Table 5 Oxidative stress Biomarkers with MSP2 alleles

\begin{tabular}{|c|c|c|c|c|}
\hline & $\mathrm{MDA}(\mu \mathrm{M})$ & GSH $(\mu \mathrm{M})$ & GPx (Units/ml) & CAT (U/mg prot) \\
\hline SCD Malaria (n) & 25 & 25 & 25 & 25 \\
\hline IC3D7 & $1.57 \pm 0.09$ & $0.74 \pm 0.01$ & $0.38 \pm 0.01$ & $3.67 \pm 0.29$ \\
\hline FC27 & $1.56 \pm 0.08$ & $0.75 \pm 0.01$ & $0.34 \pm 0.02$ & $3.31 \pm 0.21$ \\
\hline$P$ value ${ }^{a}$ & 0.9420 & 0.3705 & 0.0959 & 0.3298 \\
\hline \multicolumn{5}{|l|}{ Malaria } \\
\hline IC3D7 & $1.43 \pm 0.06$ & $0.75 \pm 0.01$ & $0.43 \pm 0.02$ & $2.87 \pm 0.19$ \\
\hline FC27 & $1.46 \pm 0.08$ & $0.74 \pm 0.01$ & $0.41 \pm 0.02$ & $3.25 \pm 0.31$ \\
\hline$P$ value $^{a}$ & 0.7571 & 0.6099 & 0.3689 & 0.3041 \\
\hline$P$ value ${ }^{b}$ & 0.2382 & 0.3859 & $0.0400^{* *}$ & $0.0282^{* *}$ \\
\hline$P$ value $^{c}$ & 0.3813 & 0.6112 & $0.0267^{* *}$ & 0.8896 \\
\hline
\end{tabular}

MDA is malondialdehyde; GSH is reduced glutathione; GPx is Glutathione peroxidase; CAT is catalase. IC3D7 and FC27 are allele families of MSP2, *** Statistically significant. ${ }^{a}$ Between IC3D7 and FC27 in each group; ${ }^{\mathrm{b}}$ Between IC3D7 in SCD malaria and malaria groups; ${ }^{\mathrm{C}}$ Between FC27 in SCD malaria and malaria groups 
[25] and malaria [7] elevates the demand for GSH. Several authors have reported an irreversible loss of GSH especially when it acts as a de-toxicant $[26,27]$. The results obtained in this study agree with the above previous work with reduction in GSH as oxidative stress increases.

GPx is an important enzymatic antioxidant found in the cytosolic and mitochondrial as well as in the peroxisomal compartments in some cells [28]. GPx plays a vital role in the elimination of both hydrogen peroxide and lipid type of peroxides. Several authors attribute the total elimination of hydrogen peroxide to GPx [28, 29]. Several other authors also report reduced GPx activity in erythrocytes under oxidative stress which is in agreement with the findings in this work where activity of GPx in malaria positive isolates is lower than the controls with the activity.

Catalase plays a vital role in the decomposition of hydrogen peroxide to water and oxygen [30]. In this study there is a significant decrease in catalase activity in the malaria positive isolates compared to controls which is in total agreement with them. Furthermore, this study also reports a further decrease activity of catalase in isolates with SCD disease than the healthy normal controls. Similar to previous studies, several authors have reported a decrease in the activity of erythrocyte catalase under oxidative stress [30,31].

In the current study, there was a non-significant trend of a lower parasitemia in SCD malaria compared to malaria patients without SCD. This may be attributed to the enhanced phagocytosis of ring-parasitized SCD RBC compared to normal RBCs [32]. This is consistent with previous study in Dar-es-Salaam, Tanzania [33]. This finding may be interpreted by the fact that greater oxidative stress (higher MDA levels) damages erythrocytes and may also directly or indirectly damage the parasites resulting in lower parasitemia. Another explanation may be that MDA leads to ineffective erythropoiesis. Furthermore, parasite density had positive correlation with MDA but this was not statistically significant. This finding may be explained by the fact that MDA is not the only end product of the destructive effects of ROS but also 4-hydroxynonenal [34]. In addition, no significant correlations were found between parasite density and MDA with other biomarkers of oxidative stress. Several components of the host enzymatic and nonenzymatic antioxidant cellular defense system are synergistically involved in protecting biological systems from ROS [7].

In this study, the prevalence of the IC3D7 was higher than the FC27 allelic family as prevoiusly reported in malaria patients without SCD [35, 36]. However, findings in this study indicate a lower MOI than previously shown in Arua, Uganda [37] and Gezira state Sudan [38] though in consistent with that in Malaysia [39]. In
Uganda, several genotypic studies of $P$. falciparum have been done in malaria patients with normal hemoglobin and sickle cell trait. A previous study in Western Uganda found no significant influence of sickle cell trait on the distribution of FC27 and IC3D7 alleic famillies [40].

Of note, no studies have investigated genetic diversity of P.falciparum strains in SCD patients with malaria in Uganda. Using a far more polymophic marker MSP2, this study showed predominance of IC3D7 family but lower MOI and no association with biomarkers of oxidative stress. Recently, a significant disparity in MSP1 genotypes and allele frequencies (K1, 91.8\%, MAD20, 32.4\%; RO33, 18.9\%) were found in SCD patients with malaria in Nigeria [13]. The lower MOI may be due to severe oxidative stress in SCD malaria. In constrast, a much lower overall MOI of 1.43 was found SCD patients with malaria in Nigeria [13]. These findings could also explained that oxidatives stress damages not only erythrocytes but also malaria parasites. Furthermore, the study found no significant differences were observed between MDA, GSH, GPx and CAT between IC3D7 and FC27 in SCD malaria and malaria samples. It can be concluded that infection by P.falciparum causes oxidative stress regardless of parasite strain.

This study had several limitations. First the study had a small sample size for each of the four groups $(\sim 50$ each) which may have limted our power to identify certain effects. Secondly, TBARS method assay is known to have limitations in that it measures many different aldehydes and decomposition products from $\mathrm{H} 2 \mathrm{O} 2$ which may translate into problems with specificity and variability of data. Thirdly, reticulocyte counts were not determined which could have provided useful information for preliminary assessment of hemolytic or dyserythropoietic anemia. More studies are needed to investigate these aspects. Nevertheless, our study provides novel insights into the effects of malaria on oxidative stress in SCD patients (HbSS).

\section{Conclusion}

This study showed increased lipid peroxidation and reduced antioxidant levels in the $\mathrm{SCD}+/$ malaria isolates than the SCD controls. Although not significantly different, the mean GMPD was lower in SCD+/ malaria patients than SCD-/malaria patients. Thus, erythrocytes from SCD patients are subjected to a double oxidative stress, the first stress due to the mutation and secondly stress induced by the developing P.falciparum parasites [32]. These results confirm severe oxidative stress in SCD patients with uncomplicated P.falciparum malaria.

\section{Abbreviations}

CAT: Catalase; DTNB: 2-nitrobenzoic acid; GMPD: Geometric mean parasite density; GPX: Glutathione peroxidase; GSH: Reduced glutathione; HbAA: Normal hemoglobin; HbAS: Sickle cell trait; HbSS: Sickle cell patients; 
MDA: Malondialdehyde; MSP2: merozoite surface protein 2; RNS: Reactive nitrogen species; ROS: Reactive oxygen species; SCD: Sickle cell disease; SD: Standard deviation; TBA: Thiobarbituric acid; TCA: Trichloroacetic acetic acid; TNB: 5'-thio-2-nitrobenzoic acid

\section{Acknowledgements}

The authors would like to thank all patients, parents and guardians who accepted to take part in the study. The authors acknowledge the medical staff at the Sickle cell Clinic, Mulago National referral Hospital and Staff at the Department of Biochemistry, College of Natural Sciences, Makerere University.

\section{Authors' contributions}

SMA and DMK designed the study. NL was BSC student and helped in recruitment of patients. SMA undertook this work as part of his MSC Research Project. SMA and DMK analyzed the data. The study was undertaken under the supervision of DMK. All authors read and approved the final manuscript

\section{Authors' information}

SMA (BSC, MSc Biochemistry) was an MSc Biochemistry Student at Department of Biochemistry and Sports Science, School of Biosciences, College of Natural Sciences.

$\mathrm{NL}$ (BSC) was a BSC student at Department of Biochemistry and Sports Science, School of Biosciences, College of Natural Sciences.

DMK (BSC, MSc Molecular Biology, PhD) is Lecturer at Department of Biochemistry and Sports Science, School of Biosciences, College of Natural Sciences, Makerere University.

\section{Funding}

The research received financial support from the DRGT 377 Project of SIDA Phase IV the Makerere-Swedish Bilateral Programme (2015-2020) and Embassy of Belgium. The funding bodies did not have any involvement in the design of this study or in the collection, analysis, and interpretation of data or in writing of the manuscript.

\section{Availability of data and materials}

The datasets used and/or analyzed during the current study are available from the corresponding author on reasonable request.

\section{Ethics approval and consent to participate}

All patients read and signed an informed consent form. The study was approved by the Mulago National Referral Hospital Ethics Committee and the Uganda National Council of Science and Technology.

\section{Consent for publication}

Not applicable

\section{Competing interests}

The authors declare that they are no competing interests.

Received: 18 September 2018 Accepted: 26 June 2019

Published online: 09 July 2019

\section{References}

1. WHO. Sickle-cell disease: a strategy for the WHO African region. Report AFR/ RC60/8. Geneva: World Health Organization; 2010.

2. Ndeezi G, Kiyaga C, Hernandez AG, Munube D, Howard TA, Ssewanyana I, Nsungwa J, Kiguli S, Ndugwa CM, Ware RE, Aceng JR. Burden of sickle cell trait and disease in the Uganda sickle surveillance study (US3): a cross sectional study. Lancet Glob Health. 2016;4:e195-200.

3. Piel FB, Hay SI, Gupta S, et al. Global burden of sickle cell anaemia in children under five, 2010-2050: modelling based on demographics, excess mortality, and interventions. PLoS Med. 2013;10:e1001484.

4. World Health Organization World Malaria Report. Geneva: WHO; 2014.

5. Aslan M, Thornley BD, Freeman BA. Reactive species in sickle cell disease. Ann N Y Acad Sci. 2000;899:375-91.

6. Atamna $\mathrm{H}$, Ginsburg $\mathrm{H}$. Origin of reactive oxygen species in erythrocytes infected with Plasmodium falciparum. Mol Biochem Parasitol. 1993;61:231-4.
7. Becker K, Tilley L, Vennerstrom JL, Roberts D, Rogerson S, Ginsburg H. Oxidative stress in malaria parasite-infected erythrocytes: host-parasite interactions. Int J Parasitol. 2004;34:163-89.

8. Percário S, Moreira DR, Gomes BA, Ferreira ME, Gonçalves AC, Laurindo PS, Vilhena TC, Dolabela MF, Green MD. Oxidative stress in malaria. Int J Mol Sci. 2012;13(12):16346-72.

9. Nur E, Biemond BJ, Otten HM, Brandjes DP, Schnog JJ; CURAMA Study Group. Oxidative stress in sickle cell disease; pathophysiology and potential implications for disease management. Am J Hematol. 2011;86(6):484-9. https://doi.org/10.1002/ajh.22012

10. Aslan M, Ryan TM, Adler B. Oxygen radical inhibition of nitric oxidedependent vascular function in sickle cell disease. Proc Natl Acad Sci. 2001; 98:15215-20.

11. Ama Moor VJ, Piema CA, Chemegne BC, Manonji H, Njinkio Noon BL, Mamiafo CT, Moukette BM, Nzufo FT, Tazoacha A. Oxidative profile of sickle cell patients in Cameroonian urban hospital. BMC Clin Pathol. 2016;10(1186).

12. Snounou G, Zhutt X, Siripoon N, Jarra W, Thaithong S, Brown N, Viriyakosol $\mathrm{S}$. Biased distribution of msp1 and msp2 allelic variants in Plasmodium falciparum populations in Thailand. Trans R Soc Trop Med Hyg. 1999;93(4): 369-74.

13. Bamidele Al, Oluwadun A, Olugbenga AA, Senapon OI. Plasmodium falciparum Merozoite surface Protein-1 polymorphisms among asymptomatic sickle cell Anemia patients in Nigeria. Acta Med Austriaca. 2016;54(1):44-53.

14. Gutteridge JMC, Stocks J, Dormandy TL. Thiobarbituric acid-reactive substances derived from autoxiding linoleic and linolenic acids. Anal Chim Acta. 1974;70:107-11.

15. Tietze F. Enzymatic method for quantitative determination of Nano gram amounts of total and oxidised glutathione. Anal Biochem. 1969;27:502-22.

16. Ursini F, Maiorino M, Gregolin C. The selenoenzyme phospholipid hydroperoxide glutathione peroxidase. Biochim Biophys Acta. 1985;839: 62-70.

17. Sinha A. Colorimetric assay of catalase. Anal Biochem. 1972;47:389-94.

18. Atroosh WM, Al-Mekhlafi HM, Mahdy MA, Saif-Ali R, Al-Mekhlafi AM, Surin J. Genetic diversity of Plasmodium falciparum isolates from Pahang, Malaysia based on MSP-1 and MSP-2 genes. Parasit Vectors. 2011 Dec 13;4:233. https://doi.org/10.1186/1756-3305-4-233.

19. Hussein M, Kassa M, Assefa A, Tadesse M, Kebede A. Genetic polymorphism of the merozoite surface protein-2 (MSP2) in Plasmodium falciparum isolates from Pawe District, north West Ethiopia. journal.pone. 2017; 10.1371/ 0177559.

20. Hebbel RP, Morgan WT, Eaton JW, Hedlund BE. Accelerated autoxidation and heme loss due to instability of sickle hemoglobin. Proc Natl Acad Sci. 1988:85:237 24.

21. Uzunova W, Pan W, Galkin O, Vekilov PG. Free heme and the polymerization of sickle cell hemoglobin. Biophys J. 2010;10:1016.

22. Del Rio D, Stewart AJ, Pellegrini N. A review of recent studies on malondialdehyde as toxic molecule and biological marker of oxidative stress. Nutr Metab Cardiovasc Dis. 2005;15(4):316-28.

23. Esan AJ. Evaluation of cortisol, malondialdehyde, blood glucose and lipid status on Haemoglobin variants in malaria infected and non - malaria infected individual. Int J Hematol Disord. 2015;10:12691.

24. Setty BN, Stuart MJ. Vascular cell adhesion molecule-1 is involved in mediating hypoxia-induced sickle red blood cell adherence to endothelium: potential role in sickle cell disease. Blood. 1996;88:2311-20.

25. Walter PB, Fung EB, Killilea DW, Jiang Q, Hudes M, Madden J, Porter J, Evans $P$, Vichinsky E, Harmatz P. Oxidative stress and inflammation in ironoverloaded patients with beta-thalassaemia or sickle cell disease. $\mathrm{Br} J$ Haematol. 2006;135(2):254-63.

26. Agil A, Sadrzadeh SMH. Hydroxy-urea protects erythrocytes against oxidative damage. Redox Rep. 2000;5(1):29-34.

27. Elias DBD, Freitas RM, Gonçalves RP, Magalhães HYF, Sousa JH, Magalhães SMM. Evaluation of the concentration of malondialdehyde and nitrite in patients with sickle cell anemia treated or not with hydroxyurea. Einstein. 2010;8(4 Pt 1):414-8.

28. Reid M, Asha B, Terrence F, Farook J. In vivo rates of erythrocyte glutathione synthesis in adults with sickle cell disease. Am J Physiol. 2006. https://doi. org/10.1152/ajpendo.00287.2005.

29. Postma NS, Mommers EC, Eling WMC, Zuidema J. Oxidative stress in malaria; implications for prevention and therapy. Pharm World Sci. 1996; 18(4):121-9. 
30. Edith L, Joseph L, Diane EH. Glutathione Peroxidase-1 in health and disease: from molecular mechanisms to therapeutic opportunities. Antioxid Redox Signal. 1995; 10.1089/ 3586.

31. Zorica AS, Nadica M, Ayse E, Daniel P, Saso B, Sonja D, Vladimir G, Aleksandar S, Onur E, Ahmet S, Ahmet A, Aleksandar JD. Glutathione peroxidase 1 (GPX1) genetic polymorphism, erythrocyte GPX activity, and prostate cancer risk. Diabetes. 2008; 11255-008.

32. Noichri Y, Chalghoum A, Chkioua L, Baudin B, Ernez S, Ferchichi S, Miled A. Low erythrocyte catalase enzyme activity is correlated with high serum total homocysteine levels in Tunisian patients with acute myocardial infarction. Diagn Pathol. 2013:1746-1596-8-68.

33. Ayi K, Turrini F, Piga A, Arese P. Enhanced phagocytosis of ring-parasitized mutant erythrocytes: a common mechanism that may explain protection against falciparum malaria in sickle trait and beta-thalassemia trait. Blood. 2004;104(10):3364-71.

34. Kumar $\mathrm{S}$, Bandyopadhyay $\mathrm{U}$. Free heme toxicity and its detoxification systems in human. Toxicol.Lett. 2005;157:175-88.

35. Chirico EN, Pialoux V. Role of oxidative stress in the pathogenesis of sickle cell disease. IUBMB Life. 2011. https://doi.org/10.1002/iub.584.

36. Kyabayinze DJ, Karamagi C, Kiggundu M, Kamya MR, Wabwire-Mangen F, Kironde F, Talisuna A. Multiplicity of Plasmodium falciparum infection predicts antimalarial treatment outcome in Ugandan children. Afr Health Sci. 2008;8(4):200-5.

37. Cattamanchi A, Kyabayinze D, Hubbard A, Rosenthal PJ, Dorsey G. Distinguishing recrudescence from reinfection in a longitudinal antimalarial drug efficacy study: comparison of results based on genotyping of msp-1, msp-2, and glurp. Am J Trop Med Hyg. 2003;68(2):133-9.

38. Mahdi Abdel Hamid M, Elamin AF, Albsheer MMA, Abdalla AAA, Mahgoub NS, Mustafa SO, Muneer MSE, Amin M. Multiplicity of infection and genetic diversity of Plasmodium falciparum isolates from patients with uncomplicated and severe malaria in Gezira state, Sudan. Parasit Vectors. 2016;9:362.

39. Mohd Abd Razak MR, Sastu UR, Norahmad NA, Abdul-Karim A, Muhammad A, Muniandy PK, et al. Genetic diversity of Plasmodium falciparum populations in malaria declining areas of Sabah, East Malaysia. PLoS One. 2016;11(3):e0152415.

40. Kiwanuka GN, Joshi H, Isharaza WK, Eschrich K. Dynamics of Plasmodium falciparum alleles in children with normal haemoglobin and with sickle cell trait in western Uganda. Trans R Soc Trop Med Hyg. 2009;103(1):87-94.

\section{Publisher's Note}

Springer Nature remains neutral with regard to jurisdictional claims in published maps and institutional affiliations.

Ready to submit your research? Choose BMC and benefit from:

- fast, convenient online submission

- thorough peer review by experienced researchers in your field

- rapid publication on acceptance

- support for research data, including large and complex data types

- gold Open Access which fosters wider collaboration and increased citations

- maximum visibility for your research: over $100 \mathrm{M}$ website views per year

At $\mathrm{BMC}$, research is always in progress.

Learn more biomedcentral.com/submissions 